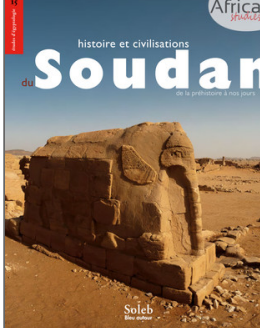

Histoire et civilisation du Soudan

De la préhistoire à nos jours

\title{
6. Le Soudan indépendant
}

\section{Bernard François}

DOI : 10.4000/books.africae. 2912

Éditeur : Africae, Soleb, Bleu autour

Lieu d'édition : Paris, Khartoum

Année d'édition : 2017

Date de mise en ligne : 17 janvier 2022

Collection : Africae Studies

EAN électronique : 9782493207074

\section{(2) OpenEdition}

\section{Books}

http://books.openedition.org

\section{Référence électronique}

FRANÇOIS, Bernard. 6. Le Soudan indépendant In : Histoire et civilisation du Soudan : De la préhistoire à nos jours [en ligne]. Paris, Khartoum : Africae, 2017 (généré le 28 janvier 2022). Disponible sur Internet <http://books.openedition.org/africae/2912>. ISBN : 9782493207074. DOI : https://doi.org/10.4000/ books.africae. 2912 . 


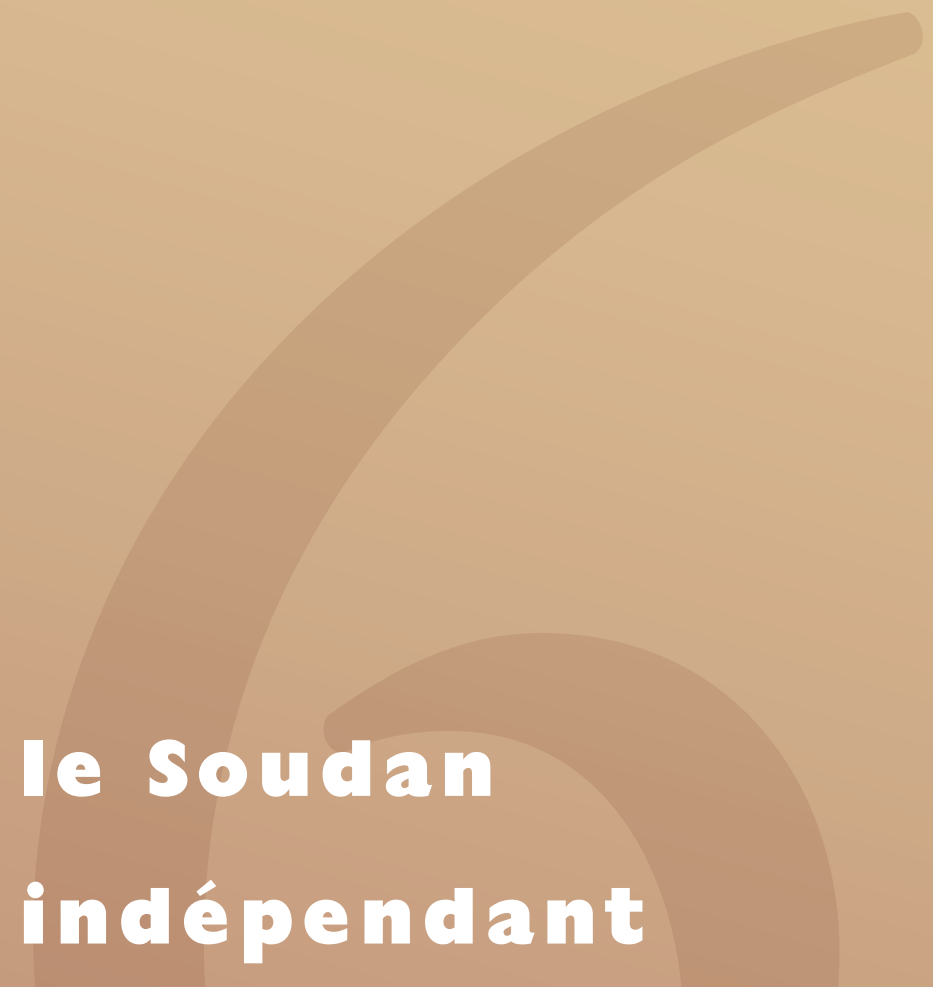


Les débuts parlementaires. Le $1^{\text {er }}$ janvier 1956, le Soudan devient officiellement indépendant. Le gouvernement de coalition d'Ismaïl al-Azhari se doit tout à la fois de développer l'économie du pays et d'établir une constitution qui reconnaisse la diversité ethnique et religieuse du Soudan, tout en exprimant la volonté et les aspirations de tous les Soudanais. Les principaux partis ne réussissent pas à s'accorder sur un agenda, et génèrent la méfiance des Sudistes, car, à l'exception des communistes, tous envisageaient une constitution basée sur l'islam.

Une mauvaise récolte de coton, en 1957, provoque une pénurie de devises étrangères et, par la suite, des restrictions. De plus, les relations avec l'Égypte se tendent et cette dernière impose un blocus sur les produits soudanais. L'Oumma remporte les élections de 1958 mais la gestion erratique et les rumeurs de corruption alimentent le mécontentement populaire.

\section{Le régime militaire du général Abboud (|958-1964)}

le Soudan

\section{4}

de 1820

à nos jours
En novembre 1958, le Premier ministre de l'Oumma, Abdullah Khalil, craignant de perdre le pouvoir, imagine de lancer un coup d'État contre son propre gouvernement, avec la complicité du chef d'état-major, le général Ibrahim Abboud. Le bras droit du général Abboud est le gendre du Premier ministre qui espére par cette voie contrôler les militaires et assurer une présidence à vie à Sayyd Abd-er-Rahman al-Mahdi. Malheureusement, un an plus tard, le gendre est mis à la retraite et Sayyd Ab-er-Rahman al-Mahdi est décédé. L’Oumma a perdu tout poids politique et les militaires contrôlent l'ensemble du pouvoir.

Seul résultat positif de cette période, l'amélioration des relations avec l'Égypte: le général Abboud signe avec l'Égypte les accords de 1959 sur le partage des eaux du Nil, faisant accepter la création du barrage de Roseires en contrepartie des terres perdues par l'agrandissement du barrage d'Assouan, mais cela lui vaut l'inimitié des habitants de la Nubie, d'autant que le gouvernement échoue à y créer les conditions favorables à son développement économique et social.

Le général Abboud va contribuer à augmenter la méfiance du Sud en y favorisant la nomination d'officiels du Nord et en y encourageant l'islam et l'arabe. En 1960, le jour de repos - jusqu'ici, au Sud, le dimanche - devient le vendredi, jour de prière des musulmans... alors que presque tous les habitants sont chrétiens ou pratiquent les religions traditionnelles. L'article 5 de la Constitution fait de l'islam la «religion d'État». Les chrétiens ne peuvent plus ouvrir d'écoles et, en 1964, les missionnaires étrangers sont expulsés (350 écoles sont reprises par l'État). 

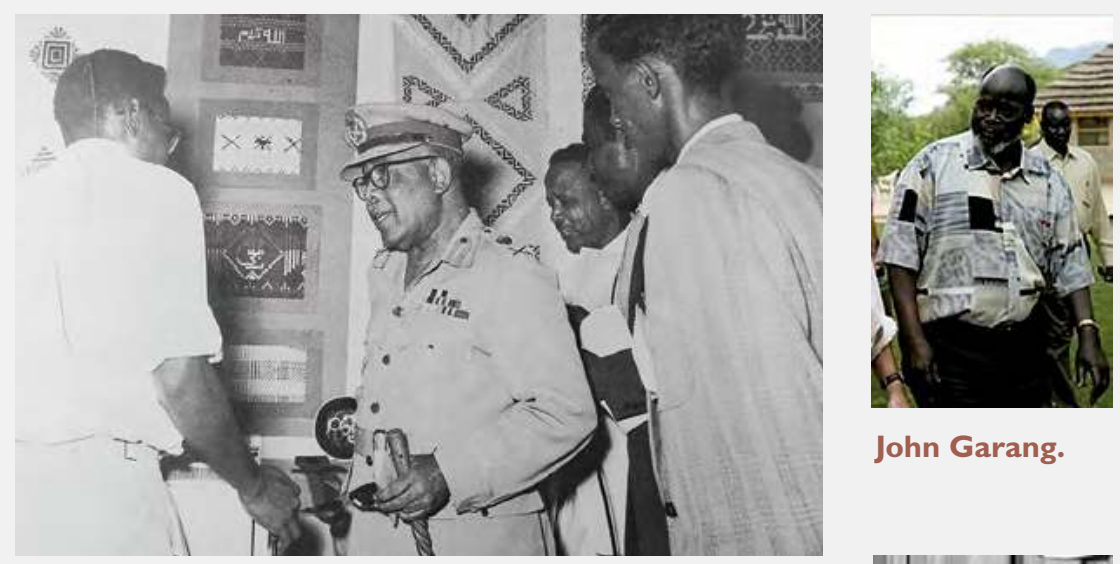

John Garang.

Le général Abboud.

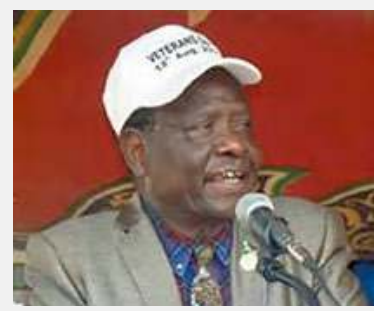

Joseph Lagu.

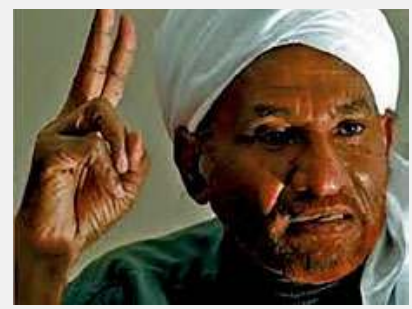

Saddiq al-Mahdi.

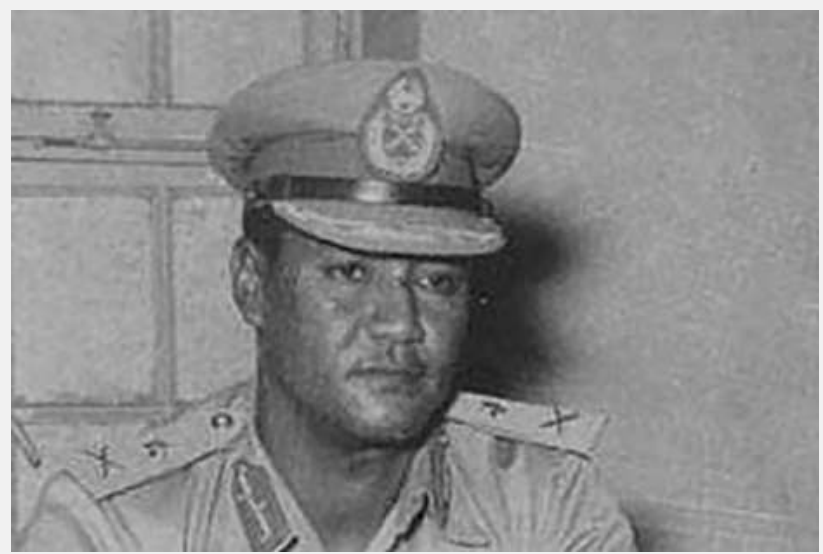

Le général Nimeiry.

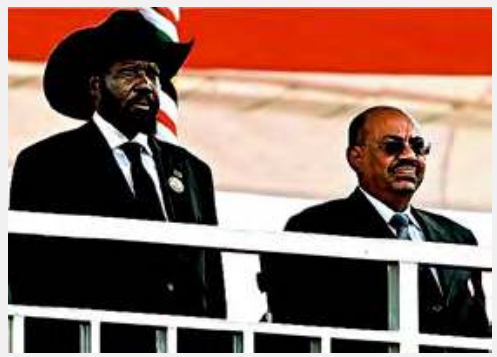

Omar el-Bechir.

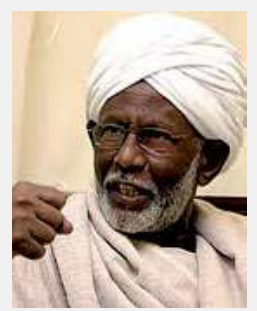

Hassan al-Turabi.

Les présidents

el-Bechir, du Soudan

(à droite), et Kiir, du Soudan

du Sud (à gauche), en 2011. 
En septembre 1963, après avoir déserté des forces armées soudanaises dont il était officier, Joseph Lagu devient le leader du mouvement nationaliste armé Anya-Nya ${ }^{1}$, qui réclame l'indépendance totale du Sud; la rébellion avait commencé dès 1955 (voir supra, p.660 sq.) mais elle ne deviendra réellement organisée qu'à partir de 1963. La Sudan African National Union (Sanu), créée hors du Soudan au début des années soixante, deviendra la voix des dizaines de milliers de réfugiés fuyant l'instabilité croissante et les combats au Sud entre l'armée et la rébellion des Anya-Nya.

Quant aux Frères musulmans, arrivés sur la scène politique à la fin des années cinquante, ils furent, comme les communistes, interdits sous le condominium, ainsi que sous le premier régime parlementaire et sous le régime militaire.

\section{Retour à la démocratie (|964-||969) le combat des chefs}

le Soudan

\section{6}

de 1820

à nos jours
Le régime militaire est renversé en octobre 1964 par un soulèvement populaire accompagné d'un vaste mouvement de grève. Les nouveaux leaders s'engagent à ne pas retomber dans les errements du premier régime parlementaire. La période 1964-1969 est dominée par l'aggravation des combats au Sud; des atrocités seront commises tant du côté gouvernemental que de celui des rebelles Anya-Nya.

Les élections d'avril 1965 ramènent au pouvoir une coalition gouvernementale issue des deux grands partis religieux, Unionistes et Oumma. Au sein de l'Oumma, le jeune Sadiq al-Mahdi, rentré d'Oxford en 1961, va s'opposer à son oncle, l'imam de la confrérie, en cherchant à transformer l'Oumma en un parti moderne capable de rallier les élites urbaines, par-delà les clivages religieux. Le différend va s'amplifier entre le neveu et l'oncle. En 1966 ce dernier mobilise les nomades Kababish et Baggara qui, venus du Kordofan, marchent sur la capitale. Finalement, le Premier ministre et chef de l'Oumma Ahmed Mahgoub est mis en minorité au Parlement, les Unionistes et les Sudistes ayant voté avec la faction rebelle de l'Oumma: à trente et un ans, Sadiq al-Mahdi devient Premier ministre.

Le problème principal devient peu à peu la guerre au Sud et Sadiq al-Mahdi, en dépit de son modernisme, y défend sa vision de la mission civilisatrice de l'islam: "L'islam a une sainte mission en Afrique et le Sud Soudan est le début de cette mission. \Il écrit au pape Paul VI en lui suggérant une coexistence dans le but de «transformer tous les païens

1 Le nom «Anya-Nya » vient du nom du venin de la vipère du Gabon dans lequel les chasseurs trempent leur flèche. 
en croyants» et en soulignant l'intérêt commun de l'islam et de la chrétienté sur ce point. Il favorise la poursuite des tentatives d'islamisation armées, ce qui le discrédite auprès des Sudistes ainsi que des libéraux du Nord. Il est mis en minorité au Parlement, et l'ancien Premier ministre revient au pouvoir à la tête d'une coalition.

Une première table ronde est organisée à Khartoum en 1965. Elle permet aux Nordistes et aux Sudistes d'exprimer les positions qui vont les diviser pendant quarante ans. Les premiers, pour qui la situation résulte de la politique coloniale et de l'hypocrisie des missionnaires chrétiens, proposent une administration régionale pour la gestion de la santé, de l'éducation, du commerce et de l'agriculture; de son côté la SANU considère que les Sudistes doivent trancher par référendum entre une union, une fédération ou l'indépendance. Une commission de douze personnalités établit la base des accords devant être signés à Addis-Abeba et permettre une suspension durable des hostilités.

En 1966, les élections ramènent le Dup et l'Oumma au pouvoir, sans que les Sudistes disposent d'une représentation parlementaire valable. En 1968, aucun parti n'atteint la majorité absolue. L'absence significative de progrès sur le front économique et sur celui de la guerre avec le Sud crée les conditions d'un nouveau coup d'État militaire.

\section{Les années Nimeiry (1969-1985)}

Le général Nimeiry — pronassérien qui a servi dans la guerre au Sud — et ses jeunes Officiers libres prennent le pouvoir par un coup d'État sans effusion de sang et installent un régime autoritaire qui va durer seize ans, grâce à sa faculté de jouer en permanence de ses différents alliés les uns contre les autres.

Sadiq al-Mahdi est immédiatement arrêté, alors que son oncle, chef de file de la faction traditionaliste de l'Oumma, se réfugie dans l'île d'Aba, berceau historique du mahdisme. Après moins d'un an, le général Nimeiry, sous la pression conservatrice, se débarrasse de son allié communiste, qui réagit par une tentative de coup d'État en juillet 1971; mais la Libye, l'Égypte et le Royaume-Uni interviennent en faveur de Nimeiry, qui va purger l'administration et l'armée des éléments de gauche.

Il tente ensuite sans succès un rapprochement avec la faction traditionaliste de l'Oumma. S'ensuit une répression militaire féroce contre les partisans de l'Oumma à Omdurman; l'île d'Aba est bombardée par des MiG égyptiens qui provoquent 1300 morts; l'imam al-Hadi lui-même est tué durant sa fuite vers la frontière éthiopienne. Ces massacres marquent la fin du mahdisme historique. Subsiste Sadiq al-Mahdi qui a pu rallier à lui les modernistes et qui quitte le pays dès sa libération de prison. De Libye, où il créa le Front National Soudanais avec Hassan al-Tourabi, chef de file 
le Soudan

\section{8}

de 1820

à nos jours de la tendance dure des Frères musulmans, il mènera trois tentatives de renversement du régime en 1970, 1975 et 1977. Après quoi Sadiq al-Mahdi changera de stratégie... et deviendra membre du comité central du parti unique de Nimeiry.

La rébellion au Sud s'accroît à mesure que Nimeiry fait parler les armes. L'Éthiopie, l'Ouganda et Israël soutiennent les rebelles et leur offrent des sanctuaires, alors que les politiciens de Khartoum défendent un Soudan uni, au sein du Moyen-Orient musulman. Abel Alier, un Dinka, est nommé ministre des Affaires du Sud, pendant que Joseph Lagu unit les militaires et les politiciens du Sud dans le South Sudan Liberation Movement qui souhaite ouvrir des pourparlers avec le gouvernement. Les accords d'Addis-Abeba, en 1972, mettent fin à la première guerre civile. Les Sudistes gagnent l'autonomie régionale; et si l'arabe reste langue nationale, l'anglais pourra être la première langue au Sud. Néanmoins, Nimeiry sort affaibli du processus de paix: il n'a pas obtenu un large soutien du Nord, qui considère qu'il a cédé aux exigences du Sud.

Durant les années qui suivent il déploie plus d'efforts pour se maintenir au pouvoir que pour bâtir un consensus national. Sa politique économique exacerbe les difficultés. Inflation, mauvaise gestion et corruption entraînent l'arrêt des appuis de la Banque mondiale en 1981. Le FMI suspend ses concours à défaut de mesures d'austérité. Et l'afflux de centaines de milliers de réfugiés fuyant la famine et la guerre qui sévissent en Éthiopie et dans la future Érythrée aggrave les difficultés du Soudan.

De surcroît, Nimeiry divise le Sud en trois régions; il prévient ainsi l'unification d'un bloc sudiste et monte les leaders les uns contre les autres. Ceci pousse les Sudistes, désillusionnés par l'absence de résultats concrets après les accords de paix de 1972, à reprendre la lutte armée. Et ce d'autant plus que Nimeiry, en 1983, nomme Hassan al-Tourabi procureur général et impose la shari'a au Soudan tout entier. La plus sérieuse défection est alors celle du colonel John Garang de Mabior dont le bataillon se soulève durant l'été 1983 - qui crée la Sudan People’s Liberation Army (SPLA).

\section{Mahmoud Mohamed Taha}

Le penseur rénovateur musulman Mahmoud Mohamed Taha, partisan d'un islam réformateur, s'élève en 1983 contre l'introduction de la shari'a comme loi fondamentale. Personnalité charismatique et très respectée du peuple soudanais, il avait publié en 1951 le livre qui exprimait son message: Al-rissala al-thaniya mina al-islam (en français : Un islam à vocation libératrice). 


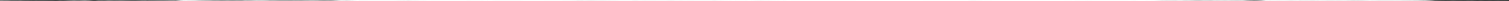


Arrêté le 5 janvier 1985, il passe en jugement deux jours plus tard. Devant le tribunal il déclare: 《J'ai affirmé à plusieurs reprises mon opinion, selon laquelle les lois de septembre 1983 bafouent la shari'a islamique et l'islam lui-même. De plus, ces lois ont défiguré la shari'a islamique et l'islam jusqu’à les rendre repoussants. Plus encore, ces lois ont été édictées et utilisées pour terroriser le peuple et le soumettre à force d'humiliation. Ces lois ont également mis en péril l'unité nationale du pays. Voilà quelles sont mes objections sur le plan théorique. Sur le plan pratique, les juges qui ont imposé ces lois n'ont pas les qualifications techniques requises. Ils ont aussi failli moralement, ne refusant pas de se placer sous le contrôle des autorités de l'exécutif, qui les ont utilisés pour bafouer les droits des citoyens, humilier le peuple, défigurer l'islam, insulter l'intellect et les intellectuels et pour humilier des opposants politiques. Pour toutes ces raisons, je n'ai pas l'intention de coopérer avec un tribunal qui a trahi l'indépendance de la justice et qui ne s'est pas opposé à ce qu'on le manipule afin d'humilier le peuple, d'insulter la libre pensée et de persécuter des opposants politiques.

Le verdict, sans appel, tombe après à peine deux heures de procès: "peine capitale par pendaison». Pourquoi? Selon ce tribunal expéditif, Mahmoud Mohamed Taha est condamné pour hérésie et pour opposition à l'application de la loi islamique. Un tribunal spécial confirme la sentence le 15 janvier et deux jours plus tard, le président Nimeiry fixe l'exécution au 18 janvier. Un hélicoptère emportera le corps dans le désert pour qu'il soit inhumé en un endroit secret.

Finalement, le régime implose sous l'effet combiné de la famine, de la crise économique, de la guerre dans le Sud et du désenchantement populaire, qui se traduit par une grève massive contre le pouvoir, alors que Nimeiry est à l'étranger et se réfugiera bientôt en Égypte.

\section{Deuxième retour à la démocratie (|985-|989)}

À l'occasion d'un voyage du Président aux États-Unis, le général Suwar al-Dahab, commandant en chef de l'armée, annonce que celle-ci a entendu la volonté du peuple. Il dissout le Parlement, révoque la constitution de 1973, crée un conseil militaire de transition et promet la fin de la guerre civile au Sud, commençant par instituer un cessez-le-feu et décréter l'amnistie. Des membres de la «société civile» rencontrent le Spla en dehors du Soudan. Mais le général Dahab ne réussit pas à obtenir le soutien du Nord pour abroger la shari'a. Au bout d'une année, il remet le pouvoir aux civils.

Sadiq al-Mahdi, dont le parti a remporté les élections, forme un gouvernement de coalition avec le Dup de Mirghani, mais la coexistence est difficile et il se rapproche bientôt du New Islamic Front (Nif) 
de Tourabi. En 1988, Mirghani rencontre John Garang et un accord de cessez-le-feu lié à la suspension de la sharía est trouvé; après une résistance initiale, Sadiq al-Mahdi accepte en mars 1989, et une nouvelle coalition est formée pour mettre en œuvre l'accord Dup-SpLA.

Sadiq al-Mahdi s'accorde avec la communauté internationale pour autoriser l'opération Lifeline Sudan qui permettra d'acheminer vivres et aide médicale dans les zones contrôlées par le SpLA et celles contrôlées par le gouvernement. Alors que l'armée le presse de négocier et qu'il pense à organiser une conférence avec les Sudistes, des forces s'opposent à toute réduction du rôle de l'islam au Soudan.

\section{Les années el-Bechir (1989-2019)}

Le 30 juin 1989, le colonel Omar Hassan el-Bechir, qu’appuie le mouvement islamiste, renverse le gouvernement par un coup d'État sans effusion de sang. Il établit un Conseil de commandement révolutionnaire pour le salut national de quinze membres (auto-dissoud en 1993) qui font allégeance à Hassan al-Tourabi. Leader des islamistes, celui-ci considère que le Soudan a un rôle à jouer dans la diffusion de l'islam dans le monde et peut soutenir les insurrections islamistes régionales; il invite ainsi Oussama Ben Laden à vivre au Soudan.

Tourabi, pour qui la victoire militaire au Sud est possible, appelle ses combattants au jihad — autrement dit à mourir en martyrs. Le nombre de victimes s'accroît des deux côtés, ce qui renforce l'opposition à cette lutte parmi les habitants du Nord.

Après une tentative d'assassinat contre le président égyptien, Hosni Moubarak - perpétrée en 1995 à Addis-Abeba par un groupe extrémiste égyptien opérant à partir du Soudan —, les pays voisins deviennent suspicieux et les Nations unies menacent le Soudan de sanctions. Oussama Ben Laden est expulsé en 1996 sous la pression des ÉtatsUnis qui, en 1993, avaient inscrit le Soudan sur la liste des pays supportant le terrorisme et qui décident de sanctions en 1997. En 1998, un missile de croisière américain annihile, à Khartoum Nord, une fabrique de produits pharmaceutiques considérée comme un possible centre de fabrication d'armes de guerre chimique.

En 1999, un changement s'opère dans l'équipe au pouvoir, dont Hassan al-Tourabi est écarté.

Au Sud, l'opposition armée est décimée par une lutte fratricide entre Riek Machar et le Spla de John Garang, ce qui provoque un exode massif de réfugiés vers Khartoum: des camps sont créés dans les alentours de la capitale soudanaise. 
le Soudan

\section{2}

de 1820

à nos jours
Plusieurs conférences internationales visent à obtenir une fin négociée du conflit. En 1997, une déclaration de principes est acceptée par Khartoum après trois ans de négociations sous l'égide de l'Igad ${ }^{1}$. Cette déclaration donne priorité à l'unité du Soudan, alors que le Sud Soudan demandait le droit à l'autodétermination. La guerre entre l'Érythrée et l'Éthiopie contribue à l'enlisement du processus.

En 2002, le Soudan accède aux attentes des Américains en matière de terrorisme, et un accord de cessez-le-feu intervient. En juillet de la même année, le protocole de Machakos pose les principes d'un accord de paix. Les négociations sous médiation kényane démarrent aussitôt et aboutissent à la signature du Comprehensive Peace Agreement (CPA) le 9 janvier 2005. Il prévoit une période de transition de six ans, au terme de laquelle doit se tenir un référendum sur l'autodétermination du Sud; et il met en place pour cette période un gouvernement d'union nationale, dont John Garang devient le premier vice-président.

Le nouveau gouvernement doit faire face à la rébellion du Darfour, qui s'est déclenchée en 2003. La répression se traduira par des dizaines de milliers de morts et le déplacement de près de deux millions de personnes. L'utilisation par le gouvernement de milices armées (Janjawid) qui se rendent coupables d'atteintes aux droits de l'homme est condamnée par la communauté internationale. Le gouvernement est aussi confronté au «mahdisme pour la justice et l'égalité» dont se revendiquent certains partis. De plus, à partir de 2005 - et malgré une tentative du Tchad de s'impliquer comme facilitateur de la paix entre les factions au Darfour —, ce pays et le Soudan seront en état de belligérance jusqu'en janvier 2010. En 2006, un accord de paix met fin à une autre rébellion, dans les provinces de l'est.

Les négociations prévues par la CPA vont prendre un retard important. Les élections sont organisées in extremis. Bien que ne répondant pas à tous les critères de régularité, ces élections sont reconnues par la communauté internationale, et vont confirmer, tant au Soudan qu'au Sud Soudan, la prééminence de chacun des deux partis au pouvoir, le NcP et le SPLM, laissant ici et là une opposition frustrée. Le désistement du candidat du Splm au Nord, sous la pression de son propre parti, est un signe de la collaboration entre ces deux forces dominantes. Le référendum, qui constitue le dernier point majeur du CPA, est organisé dans le respect des délais prévus, le 4 janvier 2011, et le Nord en accepte le résultat: un raz-de-marée en faveur de la sécession - alors que le régime de Khartoum a très longtemps cru que l'unité serait confirmée, ce qui correspondait aux souhaits 
de la population du Nord pour qui la séparation a été vécue comme un traumatisme. Le 9 juillet 2011, l'indépendance du Soudan du Sud est proclamée — dans l'euphorie au Sud —, après plus de trente ans de guerre civile.

Des nuages vont s'amonceler dès après la tenue du référendum. Les questions non résolues vont ternir la situation: la délimitation des frontières en est restée à de timides débuts; le partage des richesses, à commencer par celui du pétrole, n’a pas été sereinement effectué; la question de la citoyenneté, surtout celle des centaines de milliers de Soudanais du Sud qui vivent à Khartoum et dans le Nord depuis des décennies, n’a pas trouvé de solution; le sort des troupes du SPLM cantonnées dans les monts Noubas n'est pas réglé, et les consultations populaires dans les deux provinces du Sud Kordofan et du Nil Bleu ont été retardées maintes fois... C'est d'abord le sort de la ville d'Abyei qui va provoquer de graves incidents.

\section{La transition démocratique}

Mais ce sont les difficultés économiques qui, après trente ans de pouvoir, vont entraîner la chute du régime: privé du pétrole du Sud et dépendant de l'aide des pays du Golfe et de l'Arabie saoudite, celui-ci consacre une part démesurée de son budget aux «services de sécurité». Les pénuries s'accumulent: essence, fuel, gaz, blé, farine, pain, sucre et de nombreux produits de première nécessité disparaissent peu à peu des étals. Déclenchées par un triplement des prix du pain, les manifestations commencent, le 19 décembre 2018, dans la ville d'Atbara et s'étendent à d'autres villes avant de gagner Khartoum. L'Association des professionnels du Soudan (SPA) sort de la clandestinité et organise le mouvement. Le 4 avril 2019, une marée humaine s'installe devant le quartier général de l'armée à Khartoum. Une semaine plus tard, le 11, Omar el-Bechir est renversé par un groupe de généraux qui forment un Conseil militaire de transition (Смт) dirigé par le général Abdel Fattah al-Bourhane. Le 3 juin, le sit-in des manifestants réclamant «tout le pouvoir aux civils» est dispersé dans le sang: au moins 127 morts.

À la suite de la médiation de l'Éthiopie et de l'Union africaine, les généraux font des concessions et une "déclaration constitutionnelle», encadrant la transition, est signée le 17 août par Mohamed Daglo, pour le CMT, et Ahmed al-Rabie, pour l'Alliance pour la liberté et le changement (ALC, dont fait partie la SPA) : un Conseil souverain de onze membres — six civils et cinq militaires - dirigera le pays pendant une période de transition de trois ans et une Assemblée législative de trois cents membres sera mise en place. Ce même jour s'est ouvert à Khartoum le procès "pour corruption" de l'ex-président. Le 21 août, Abdallah Hamdok, ex-économiste à l'ONU, a été investi Premier ministre du Soudan et le CMT a été dissous - 